https://doi.org/10.29013/ESR-19-9.10-42-44

Murodov Jurabek Norpulatovich, independent researcher,

Tashkent state Technical University

E-mail:shodlik29081986@mail.ru

\title{
SOURCES AND METHODS FOR CALCULATING UNCERTAINTY OF MEASUREMENTS
}

Abstract. The article considers the types and contents of the standard uncertainty of the measurement results. It also shows the main steps in calculating extended uncertainty. Recommendations are given for determining the coverage rate.

Keywords: measurement uncertainty, measurement result, sources of uncertainty, reference value, measuring instruments, expanded uncertainty.

There are various sources of measurement uncertainty, including:

- incomplete determination of the measured value;

- imperfect implementation of the definition of the measured value;

- a non-representative sample - the measured sample may not represent a definable measurable quantity;

- Inadequate knowledge of the effects of environmental conditions affecting the measurement, or imperfect measurement of environmental conditions;

- subjective systematic error of the operator when taking readings of analog measuring instruments;

- final resolution of measuring instruments or threshold of sensitivity;

- inaccurate values attributed to the standards used for measurement and standard samples of substances and materials;

- Inaccurate values of constants and other parameters obtained from external sources and used in the data processing algorithm;

An unknown systematic effect cannot be taken into account in estimating the uncertainty of a measurement result, but it contributes to its error.

The components of uncertainty are grouped into two categories in accordance with their assessment methods "A" and "B". These categories relate to uncertainty and are not a substitute for the words "random" and "systematic".

The estimated variance $u 2$, which characterizes the uncertainty component obtained as a result of type A estimation, is calculated from the series of repeated observations, and it is a familiar statistical estimate of the variance $s 2$. The estimated standard deviation $\mathrm{u}$, which is the positive square root of $u 2$, is thus the standard deviation of $s$, and it is sometimes called the standard uncertainty of type A for convenience. For the component of the uncertainty obtained from the type $B$ estimation, the estimated variance $u 2$ is calculated, using the available data and the estimated standard deviation $\mathrm{u}$, and sometimes called the standard uncertainty of type $B$.

Thus, the standard uncertainty of type $A$ is obtained from the probability density function obtained from the observed frequency distribution, while the standard uncertainty of type $B$ is obtained from the estimated probability density function based on the degree of confidence that the event will happen (this probability is often called subjective probability).

NOTE An estimate of the type B uncertainty component is generally based on a relatively reliable information fund. 
The standard uncertainty of the measurement result, when the result is obtained from the values of a number of other quantities, is called the total standard uncertainty and is denoted as uc. It is the estimated standard deviation associated with the result and is equal to the positive square root of the total variance obtained from all components of the variance and covariance.

To satisfy the requirements in some areas of industry and commerce, as well as the requirements in the field of health and safety, the expanded uncertainty $U$ is obtained by multiplying the total standard uncertainty uc by the coverage factor $k$. The value $U$ shows the interval near the measurement result, within which, as you might expect, there is a large part of the distribution of values that could be reasonably attributed to the measured value.

The coverage factor should always be specified so that, if necessary, the standard uncertainty can be obtained again.

If all the quantities on which the measurement result depends are changing, their uncertainty can be estimated by statistical means. However, since in practice this is rarely possible due to limited time and resources, the uncertainty of the measurement result is usually estimated using the mathematical measurement model and the law of distribution of uncertainty.

Since the mathematical model may be incomplete, all the values mentioned should be changed to the fullest practical degree, so that the estimation of uncertainty, as far as possible, could be based on the observed data. The mathematical model should be reviewed whenever the observed data, including the results of independent determinations of the same magnitude, indicate that the model is incomplete. A well-designed experiment can significantly improve the reliability of uncertainty estimates and is part of the art of making measurements.

In order to decide whether the measuring system is functioning normally, the experimentally observed variability of its output values, estimated by their observed standard deviations, we can compare it with the predicted standard deviation obtained by summing the various uncertainty components that characterize the measurement. In such cases, only those components should be considered that can contribute to the experimentally observed variability of the output quantities.

In some cases, it is not necessary to include the uncertainty of the correction for a systematic effect in the estimation of the uncertainty of the measurement result.

1. Express mathematically the relationship between the measured value of $Y$ and the input quantities $\mathrm{Xi}$, on which it depends:

$$
Y=f\left(X_{1}, X_{2}, \ldots, X_{n}\right) \text {. }
$$

The function $f\left(X_{i}\right)$ must contain every value, including all corrections and correction factors, which can add a significant component to the uncertainty of the measurement result.

2. Determine $x i$ - the estimated value of the input quantity $X_{i}$, either based on statistical analysis of the series of observations or other means.

3. Estimate the standard uncertainty $\mathrm{u}\left(X_{i}\right)$, of each input estimate xi. For the input estimate obtained from the statistical analysis of the series of observations, the standard uncertainty is estimated by type A. For the input estimate obtained by other means, the standard uncertainty is estimated by type $B$.

4. If the values of any input values are correlated, evaluate their covariance.

5. Calculate the measurement result, ie estimate $y$ of the measured quantity $Y$ from the functional dependence $f(X i)$, using the estimates xi obtained in step 2 for the input quantities $X i$.

6. The total standard uncertainty $u c(y)$ of the measurement result $y$ is determined from the standard uncertainties and covariances associated with the input estimates. If the measurement procedure allows you to simultaneously determine more than one input quantity, calculate their covariance.

7. If you want to give expanded uncertainty $U$, whose goal is to provide an interval from $(y-U)$ to $(y+U)$, within which, presumably, there is a large 
part of the distribution of values that can reasonably be attributed to the measured value of $Y$, multiply the total standard uncertainty $u c(y)$ per coverage factor $k$, usually in the range of 2 to 3 , to obtain

$$
U=k \cdot u c(y) \text {. }
$$

The coefficient $k$ is selected based on the desired level of confidence required for the interval.

8. The y measurement result is written together with its total uncertainty $u c(y)$ or extended uncertainty $U$. Describe how y and $u c(y)$ are obtained.

\section{References:}

1. Zakharov I. P., Kukush V.D. Theory of uncertainty in measurements. Textbook: - Kharkov, Consum, 2002. - $256 \mathrm{p}$.

2. RMG 91-2009. State system for ensuring the uniformity of measurements. The joint use of the concepts of "measurement error" and "measurement uncertainty".

3. COOMET R/GM/21:2011. Use of the concepts "measurement uncertainty" and "measurement uncertainty". General principles (use of concepts "error of measurement" and "uncertainty of measurement". General principles (Approved at the $21^{\text {st }}$ meeting of the COOMET Committee (April 27-28, 2011, Yerevan, Armenia). 